\title{
Computing hypergraph width measures exactly
}

\author{
Lukas Moll \\ Humboldt Universität zu Berlin \\ Berlin, Germany \\ Siamak Tazari*
Massachusetts Institute of Technology
Cambridge, USA \\ Marc Thurley ${ }^{\dagger}$ \\ Centre de Recerca Matemàtica \\ Bellaterra, Spain
}

August 9, 2021

\begin{abstract}
Hypergraph width measures are a class of hypergraph invariants important in studying the complexity of constraint satisfaction problems (CSPs). We present a general exact exponential algorithm for a large variety of these measures. A connection between these and tree decompositions is established. This enables us to almost seamlessly adapt the combinatorial and algorithmic results known for tree decompositions of graphs to the case of hypergraphs and obtain fast exact algorithms.

As a consequence, we provide algorithms which, given a hypergraph $H$ on $n$ vertices and $m$ hyperedges, compute the generalized hypertree-width of $H$ in time $O^{*}\left(2^{n}\right)$ and compute the fractional hypertree-width of $H$ in time $O\left(1.734601^{n} \cdot m\right)$. 1
\end{abstract}

Keywords: generalized hypertree-width, fractional hypertree-width, exact exponential algorithms, monotone $f$-width

\section{Introduction}

Hypergraph width measures form a class of hypergraph invariants which play an important role in studying the complexity of constraint satisfaction problems (CSPs). For a set of variables $V$, a domain $D$ and a set $C$ of constraints these problems ask for an assignment of values in $D$ to the variables such that each constraint is satisfied. This forms a generic framework for many import combinatorial problems. Therefore, quite unsurprisingly, constraint satisfaction problems are generally NP-hard. In order to obtain a more detailed picture of the complexity of these problems, there are at least two common directions to follow. One of these is the restriction of the type of constraints allowed (see, for example [Sch78, FV98, Bul06, BK09]).

\footnotetext{
*supported by a fellowship within the Postdoc-Programme of the German Academic Exchange Service (DAAD).

${ }^{\dagger}$ supported in part by Marie Curie Intra-European Fellowship 271959 at the Centre de Recerca Matemàtica, Bellaterra, Spain

${ }^{1}$ We follow usual practice in omitting factors polynomial in $n$ each time the base of the exponent is rounded. Recall that this is justified as $c^{n} \cdot n^{O(1)}=O\left((c+\epsilon)^{n}\right)$ for every $\epsilon>0$. In all other situations we use the notation $O^{*}$ which suppresses polynomial factors
} 
The second direction is the restriction of the structure which constraints impose on the variables. With a strong motivational background in database theory, this kind of restrictions forms the origin of hypergraph width measures [GLS02, GM06, Mar09b, Mar10]. The hypergraph of an instance $(V, D, C)$ of a constraint satisfaction problem has vertex set $V$ and contains for each constraint a hyperedge with the variables occurring in this constraint. In this way we can give a precise meaning to the restriction of the structure. For some class $\mathcal{H}$ of hypergraphs, the input is restricted to instances whose hypergraphs are contained in $\mathcal{H}$.

Let $\operatorname{CSP}(\mathcal{H})$ denote the constraint satisfaction problem restricted as described above. Hypergraph width measures allow for identification of tractable variants of this problem. In the case of bounded arities - that is, when the cardinality of the hyperedges is bounded by a constant - it turns out that bounded tree-width completely describes this setting, as then $\operatorname{CSP}(\mathcal{H})$ is polynomial time computable if and only if $\mathcal{H}$ has bounded tree-width [GSS01, Gro07] $2^{2}$

In the unbounded arity case the situation is different. Several hypergraph width measures have been identified which lead to larger classes of tractable $\operatorname{CSP}(\mathcal{H})$. We have here the notion of bounded (generalized) hypertree-width [GLS02] which extends bounded tree-width. Even more general are classes $\mathcal{H}$ of bounded fractional hypertree-width [GM06] which still give rise to polynomial-time computable constraint satisfaction problems.

Our Work. The central aim of the present work is an exact algorithm for fractional hypertreewidth. Note that there is a recent algorithm which approximates fractional hypertree-width [Mar09a] in polynomial time provided that it is constant. But not only is this algorithm unsuitable for large fractional hypertree-width. There is also no known non-trivial exact algorithm for this problem.

We remedy this situation by presenting an algorithm that more generally computes any hypertreewidth measure defined by some monotone width function $f$. This implies an algorithm for both fractional and generalized hypertree-width by essentially the same means. We achieve this by reducing the problem to computing a minimal triangulation of the underlying Gaifman graph of the given hypergraph. Indeed, we show that it is sufficient to compute a tree decomposition of the Gaifman graph while measuring the width of sets of vertices in the given hypergraph. This enables us to almost seamlessly adapt the combinatorial and algorithmic results known for tree decompositions of graphs to the case of hypergraphs and obtain fast exact algorithms.

Theorem 1. Let $H$ be a hypergraph on $n$ vertices and $m$ hyperedges.

(i) The generalized hypertree-width of $H$ can be computed in time $O^{*}\left(2^{n}\right)$.

(ii) The fractional hypertree-width of $H$ can be computed in time $O\left(1.734601^{n} \cdot m\right)$.

The central idea of this algorithm is the adaptation of the algorithm in [FKTV08] and the results of [FV08] to the situation of hypergraphs. All of these algorithms require exponential space in the worst case. The proof of this result is presented in Section 3 .

\section{Preliminaries}

Graphs and Hypergraphs. A hypergraph is a pair $H=(V(H), E(H))$ consisting of a set of vertices $V(H)$ and a set $E(H)$ of subsets of $V(H)$, the hyperedges of $H$. Two vertices are adjacent if there exists an edge that contains both of them. Unless otherwise mentioned, our hypergraphs have $n$

\footnotetext{
${ }^{2}$ Note that this holds under the Parameterized Complexity assumption $F P T \neq W[1]$.
} 
vertices and $m$ edges and do not contain isolated vertices (i.e. vertices which do not occur in an edge of $H$ ).

A graph is a hypergraph in which every hyperedge has cardinality 2 . Thus every concept defined for hypergraphs is also given for graphs; however, there will be some notions we will use for graphs exclusively. For a subset $U \subseteq V(G)$, we write $G[U]$ to denote the subgraph of $G$ induced by $U$. Furthermore, $G-U$ denotes the graph $G[V(G) \backslash U]$. The neighborhood of a vertex $v \in V(G)$ is $N(v)=\{u \mid\{u, v\} \in E\}$; this extends to sets of vertices by defining $N(S)=\bigcup_{v \in S} N(v) \backslash S$. A clique of $G$ is a set $C \subseteq V(G)$ such that all vertices in $C$ are pairwise adjacent in $G$. A clique is maximal if it is not properly contained in another clique. For a set $S \subseteq V$ we define $S^{2}=\{\{u, v\} \mid$ $u, v \in S, u \neq v\}$. The Gaifman graph or primal graph of a hypergraph $H$ is the graph $\underline{H}$ on $V(H)$ with $E(\underline{H}):=\{\{u, v\} \mid u, v \in e$, for some $e \in E(H)\}$.

Tree Decompositions and Width Functions. A tree decomposition of a hypergraph $H$ is a pair $(T, \mathcal{B})$, where $T$ is a tree and $\mathcal{B}=\left\{B_{t} \mid t \in V(T)\right\}$ is a family of subsets of $V(H)$, called bags, such that

(i) every vertex of $H$ appears in some bag of $\mathcal{B}$;

(ii) for every hyperedge $e \in E(H)$ there is a $t \in V(T)$ such that $e \subseteq B_{t}$; and

(iii) for every vertex $v \in V(H)$ the set of bags containing $v$ forms a subtree $T_{v}$ of $T$.

A width function on the vertex set $V$ is a monotone function $f: 2^{V} \rightarrow \mathbb{R}_{0}^{+}$, i.e. with $f(X) \leq f(Y)$ for $X \subseteq Y$. We define $\mathcal{F}(V)$ to be the set of all width functions on $V$. The $f$-width of a tree decomposition $\mathcal{T}$ is $\max \left\{f\left(B_{t}\right) \mid t \in V(T)\right\}$. The $f$-hypertree-width of a hypergraph $H$, denoted by $f$-htw $(H)$, is the minimum $f$-width of all tree decompositions of $H$. We call such a tree decomposition an $f$-optimal tree decomposition. When considering graphs, we use the analogous notion of $f$-tree-width and denote it by $f$ - $\operatorname{tw}(G)$. In this setting we obtain the tree-width of a hypergraph $H$ as follows.

Definition 1. Let $s(X)=|X|-1$; then the tree-width of $H$ is $\operatorname{tw}(H):=s-\mathrm{htw}(H)$.

Similarly, we can define other well-known width measures. Let $H$ be a hypergraph and $X \subseteq$ $V(H)$. An edge cover (w.r.t. $H$ ) of $X$ is a subset $E^{\prime} \subseteq E(H)$ such that $X \subseteq \bigcup_{e \in E^{\prime}}$ e. Define $\rho_{H}(X)$ as the size of the smallest edge cover of $X$ w.r.t. $H$. Note that this number is well-defined, as $H$ does not contain isolated vertices.

Relaxing this, we arrive at fractional edge covers. For a set $X \subseteq V(H)$ a mapping $\gamma: E(H) \rightarrow$ $[0,1]$ is a fractional edge cover of $X$ (w.r.t. $H$ ), if $\sum_{v \in e} \gamma(e) \geq 1$ for all $v \in X$. Then $\rho_{H}^{*}(X)$ is the minimum of $\sum_{e \in E(H)} \gamma(e)$ taken over all fractional edge covers of $X$ w.r.t. $H$.

Definition 2. Let $H$ be a hypergraph.

- The generalized hypertree-width of $H$ is $g h w(H):=\rho_{H^{-}} \mathrm{htw}(H)$.

- The fractional hypertree-width of $H$ is $f h w(H):=\rho_{H^{-}}^{*}$ htw $(H)$.

Separators. For two non-adjacent vertices $u, v$ of a graph $G$, a set $S \subseteq V(G)$ is a $u, v$-separator if $u$ and $v$ are in different components of $G-S$. Further, $S$ is a minimal $u, v$-separator if no proper subset of $S$ is a $u, v$-separator. Generally, $S$ is a minimal separator if it is a minimal $u, v$-separator for some $u, v$. By $\Delta_{G}$ we denote the set of all minimal separators of $G$. Observe that a minimal separator of $G$ can be contained in another one. We call minimal separators not containing another one inclusion-minimal separators and denote the set of these by $\Delta_{G}^{*}$. 

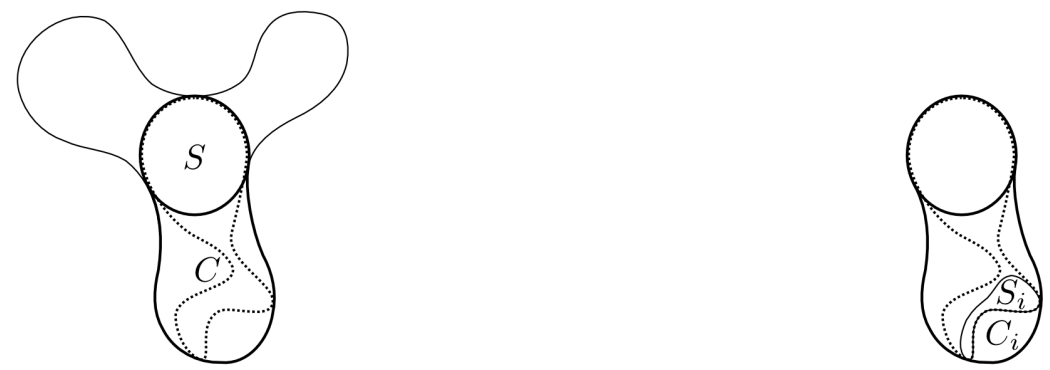

Figure 1: Left: The graph $G$ with separator $S$, component $C \in \mathcal{C}_{G}(S)$ of $G-S$ and $\Omega$ (dotted line). Right: The block (S,C) with $\Omega$ (dotted line) and the full block $\left(S_{i}, C_{i}\right)$ associated with $\Omega$ with $C_{i} \in \mathcal{C}_{G}\left(S_{i}\right) \cap \mathcal{C}_{G}(\Omega)$ and $S_{i}=N\left(C_{i}\right)$.

Let $\mathcal{C}_{G}(S)$ denote the set of connected components of $G-S$ (see Fig. 11). A component $C \in \mathcal{C}_{G}(S)$ is full w.r.t. $S$, if $N(C)=S$. By $\mathcal{C}_{G}^{*}(S)$ we denote the set of all full connected components of $G-S$. A block associated with an $S \in \Delta_{G}$ is a pair $(S, C)$ for some component $C \in \mathcal{C}_{G}(S)$. A block is called full if $C$ is full w.r.t. $S$. Note that by definition, the set $S$ of a block $(S, C)$ is required to be a minimal separator. The realization $R(S, C)$ of a block is the graph obtained from $G[S \cup C]$ by turning $S$ into a clique.

Triangulations, Potential Maximal Cliques. A graph $G$ is triangulated or chordal if every cycle of length at least 4 in $G$ has a chord, that is, an edge between two non-consecutive vertices of the cycle. A triangulation of $G$ is a chordal graph $I$ on $V(G)$ such that $E(G) \subseteq E(I)$. Furthermore, $I$ is a minimal triangulation if there is no chordal graph $I^{\prime}$ on $V(G)$ with $E(G) \subseteq E\left(I^{\prime}\right) \subset E(I)$.

A set $\Omega \subseteq V(G)$ is a potential maximal clique of $G$, if there is a minimal triangulation $I$ of $G$ such that $\Omega$ is a maximal clique in $I$. The set of all potential maximal cliques of $G$ is denoted by $\Pi_{G}$. Let $\Omega$ be a potential maximal clique with the components $\mathcal{C}(\Omega)$ of $G-\Omega$ and $C \in \mathcal{C}(\Omega)$; then $(N(C), C)$ is called a block associated with $\Omega$ (see Fig. 1).

Finally, we define the $f$-clique-number of $G$ to be $f-\omega(G):=\max _{\text {clique } \Omega}$ of $G \quad f(\Omega)$.

\section{Computing $f$-Optimal Tree Decompositions of Graphs and Hyper- graphs}

A tree decomposition $(T, \mathcal{B})$ with $\mathcal{B}=\left\{B_{t} \mid t \in V(T)\right\}$ is small, if for all $t, t^{\prime} \in V(T)$ with $t \neq t^{\prime}$ we have $B_{t} \nsubseteq B_{t^{\prime}}$. We need some well-known facts about tree decompositions:

Lemma 1. Let $G$ be a graph, $\mathcal{T}=\left(T,\left(B_{t}\right)_{t \in V(T)}\right)$ a tree decomposition of $G$, and $f \in \mathcal{F}(V(G))$ a width function. Then the following holds.

(i) For every clique $\Omega \subseteq V$ in $G$ there is a $t \in V(T)$ such that $\Omega \subseteq B_{t}$.

(ii) There is a small tree decomposition $\mathcal{T}^{\prime}$ such that $f$ - $\operatorname{width}\left(\mathcal{T}^{\prime}\right)=f$ - $\operatorname{width}(\mathcal{T})$.

(iii) For all $s, t, t^{\prime} \in V(T)$ such that $t^{\prime}$ lies on the path from $s$ to $t$ in $T$, we have $B_{s} \cap B_{t} \subseteq B_{t^{\prime}}$.

It is important to note here, that we will use the notion of $f$-htw in a slightly unusual way. Similarly to the functions $\rho_{H}$ and $\rho_{H}^{*}$, we will be interested in some width function $f_{H}$ which is defined on a hypergraph $H=(V(H), E(H))$, but then apply it to tree-decompositions of a graph $G$. The sole prerequisite is here, that $V(G)=V(H)$ to ensure that $f_{H}$ - tw $(G)$ is still well-defined. It turns out that this very concept of measuring the width of a tree decomposition of a graph using 
the width function defined on a given hypergraph is the crucial idea that makes our algorithm work in such a general form. We will make the dependence of $f$ on $H$ explicit by the subscript $f_{H}$, whenever this is important.

Lemma 2. Let $H$ be a hypergraph, $\underline{H}$ its Gaifman graph and $f_{H}$ a width function on $V(H)$. Then

$$
f_{H^{-}} \operatorname{htw}(H)=f_{H^{-}} \operatorname{tw}(\underline{H}) .
$$

In particular, $\mathcal{T}$ is a tree decomposition of $H$ if, and only if, it is a tree decomposition of $\underline{H}$.

Proof. It is easy to see that any tree decomposition of $H$ is a tree decomposition of $\underline{H}$. Conversely, the fact that any tree decomposition of $\underline{H}$ is a tree decomposition of $H$ follows from Lemma 1 (i).

Let $G$ be a graph and $\mathcal{K}_{G}$ the set of maximal cliques of $G$. The labeled tree $\mathcal{T}:=\left(T,\left(\Omega_{t}\right)_{t \in V(T)}\right)$ is a tree on $\mathcal{K}_{G}$, if every maximal clique of $\mathcal{K}_{G}$ corresponds to exactly one vertex of $T$. $\mathcal{T}$ is a clique-tree of $G$, if it satisfies the clique-intersection property:

(CI) For every pair $\Omega, \Omega^{\prime} \in \mathcal{K}_{G}$ of distinct cliques $\Omega \cap \Omega^{\prime}$ is contained in every clique on the unique path connecting $\Omega$ and $\Omega^{\prime}$ in $\mathcal{T}$.

It is well known (see e.g. Theorem 3.1 in [BP93]) that a graph $G$ is chordal if and only if it has a clique tree.

Lemma 3. Let $G$ be a chordal graph and $f \in \mathcal{F}(V(G))$ a width function. Then

$$
f-\operatorname{tw}(G)=f-\omega(G)
$$

Proof. Let $\Omega$ be a clique of $G$ that maximizes $f(\Omega)$. By Lemma 1 (i) every tree decomposition of $G$ contains a bag that contains $\Omega$. This proves $f-\operatorname{tw}(G) \geq f(\Omega)=f-\omega(G)$. To see $f-\operatorname{tw}(G) \leq$ $f-\omega(G)$, let $\mathcal{T}$ be a clique-tree of $G$. Clearly, $\mathcal{T}$ is a tree decomposition of $G$ with $f$-width $(\mathcal{T})=$ $f-\omega(G)$.

Lemma 4. Let $G$ be a graph and $f \in \mathcal{F}(V(G))$ a width function. Then

$$
f-\operatorname{tw}(G)=\min _{\substack{\text { triangulation } \\ I \text { of } G}} f-\omega(I) .
$$

Furthermore, the minimum on the right-hand side is attained by a minimal triangulation of $G$.

Proof. Let $I$ be any triangulation of $G$. Since $E(G) \subseteq E(I)$, every tree decomposition of $I$ is also a tree decomposition of $G$ and so, $f-\operatorname{tw}(G) \leq f-\operatorname{tw}(I)$. By Lemma 3 , we have thus $f$ - $\operatorname{tw}(G) \leq$ $f-\omega(I)$.

For the other direction, let $\mathcal{T}=\left(T,\left(B_{t}\right)_{t \in V(T)}\right)$ be a small $f$-optimal tree decomposition of $G$, i.e. $f-\operatorname{width}(\mathcal{T})=f-\operatorname{tw}(G)$. We construct a triangulation $I:=(V(G), E(I))$ of $G$ by transforming the vertices of every bag of $\mathcal{T}$ into a clique in $I$. That is, $E(I):=\left\{\{v, u\} \mid v \neq u, \exists t \in V(T): v, u \in B_{t}\right\}$. Obviously $\mathcal{T}$ is still a tree decomposition of $I$ with $f$ - width $(\mathcal{T})=f-\omega(I)$. We show that $I$ is chordal by arguing that $\mathcal{T}$ is a clique-tree of $I$. To see this, note that Lemma 1 (i) and the fact that $\mathcal{T}$ is small imply that there is a bijection between maximal cliques of $I$ and bags of $\mathcal{T}$. The clique-intersection property holds by Lemma1 (iii). The monotonicity of $f$ implies that the triangulation $I$ that minimizes the right-hand side of (1) can be chosen to be minimal. 


\subsection{An Algorithm to Compute the $f$-tree-width of Graphs}

The following facts about minimal separators and potential maximal cliques are well-known, see e.g. Theorem 2.10 in [KKS97] and Lemma 3.14 in [BT01]:

Lemma 5. Let $G$ be a graph, I a minimal triangulation of $G$, and $\Omega$ a potential maximal clique of $G$.

(i) Every block associated with an inclusion-minimal separator $S$ of $G$ is a full block, i.e. $\mathcal{C}_{G}(S)=$ $\mathcal{C}_{G}^{*}(S)$.

(ii) Every minimal separator of $I$ is also a minimal separator of $G$, i.e. $\Delta_{I} \subseteq \Delta_{G}$.

(iii) Every block $(S, C)$ associated to $\Omega$ is, in fact, a full block of $G$; in particular, $S \in \Delta_{G}$.

We proceed with a lemma from [KKS97]:

Lemma 6 (Lemma 3.1 in [KKS97]). Let $G$ be a graph, $S$ a minimal separator of $G$, and $I_{C}$ a minimal triangulation of $R(S, C)$ for each component $C$ of $G-S$. Then the graph $I$ on $V(G)$ with $E(I):=\bigcup_{C \in \mathcal{C}_{G}(S)} E\left(I_{C}\right)$ is a minimal triangulation of $G$.

Conversely, let $I$ be a minimal triangulation of $G$ and $S$ a minimal separator of $I$. Then $I[S \cup C]$ is a minimal triangulation of $R(S, C)$ for each component $C$ of $G-S$.

The following lemma is an extension of Theorem 3.2. in [KKS97] to our situation.

Lemma 7. Let $G$ be a non-complete graph and $f \in \mathcal{F}(V(G))$ a width function. Then

$$
f-\operatorname{tw}(G)=\min _{S \in \Delta_{G}} \max _{C \in \mathcal{C}_{G}(S)} f-\operatorname{tw}(R(S, C)) .
$$

Proof. Let $S \in \Delta_{G}$ be any minimal separator of $G$. For every component $C \in \mathcal{C}_{G}(S)$, let $I_{C}$ be a minimal triangulation of $R(S, C)$ with $f-\operatorname{tw}(R(S, C))=f-\omega\left(I_{C}\right)$ as guaranteed by Lemma 4 . By Lemma 6 the graph $I$ on $V(G)$ with $E(I):=\bigcup_{C \in \mathcal{C}_{G}(S)} E\left(I_{C}\right)$ is a minimal triangulation of $G$. By construction, there can not be an edge in $I$ connecting two different components in $\mathcal{C}_{I}(S)=\mathcal{C}_{G}(S)$; also, $S$ is a clique in $I$ and in each $I_{C}$. Thus for every clique $\Omega$ of $I$ there is a component $C \in \mathcal{C}_{G}(S)$ with $\Omega \subseteq S \cup C$ and $\Omega$ is also a clique of $I_{C}$. We have thus

$$
f-\operatorname{tw}(G) \leq f-\omega(I)=\max _{C \in \mathcal{C}_{G}(S)} f-\omega\left(I_{C}\right)=\max _{C \in \mathcal{C}_{G}(S)} f-\operatorname{tw}(R(S, C)),
$$

where the left most inequality is given by Lemma 4.

Conversely, let $I$ be a minimal triangulation of $G$ that minimizes $f-\omega(I)$ and hence $f$ - $\operatorname{tw}(G)=f-\omega(I)$ by Lemma 4 . Let $S$ be a minimal separator of $I$; by Lemma 5 (iii), we know $S \in \Delta_{G}$. By Lemma 6 , we have that $I[S \cup C]$ is a minimal triangulation of $R(S, C)$ for every component $C \in \mathcal{C}_{G}(S)$ of $G-S$. Since every clique of $I[S \cup C]$ is also a clique of $I$, we have

$$
f-\operatorname{tw}(R(S, C)) \leq f-\omega(I[S \cup C]) \leq f-\omega(I)=f-\operatorname{tw}(G) .
$$

Again, the leftmost inequality follows from Lemma 4.

Lemma 7 provides an equation for the $f$-tree-width of a graph in terms of its minimal separators. However, it would be preferable to work only with inclusion-minimal separators and full blocks. Fortunately, this can be achieved via the following lemma:

Lemma 8. Let $G$ be a non-complete graph and $f \in \mathcal{F}(V(G))$ a width function. Then

$$
f-\operatorname{tw}(G)=\min _{S \in \Delta_{G}^{*}} \max _{C \in \mathcal{C}_{G}^{*}(S)} f-\operatorname{tw}(R(S, C)) .
$$


Proof. Suppose the minimum on the right-hand side of (2) is achieved only by non-inclusion-minimal separators and let $S \in \Delta_{G}$ be such a separator. Let $S^{\prime} \subset S$ be an inclusion-minimal separator in $\Delta_{G}$. Consider a component $C \in \mathcal{C}_{G}\left(S^{\prime}\right)$; it must be that $C=S^{\prime \prime} \cup C_{1} \cup \cdots \cup C_{t}$, where $S^{\prime \prime} \subseteq S \backslash S^{\prime}$ and $C_{1}, \ldots, C_{t} \in \mathcal{C}_{G}(S)$. Let $\mathcal{T}_{i}$ be obtained from an $f$-optimal tree decomposition for $R\left(S, C_{i}\right)$, for $1 \leq i \leq t$, by removing the vertices of $S \backslash\left(S^{\prime} \cup S^{\prime \prime}\right)$ from every bag. By creating a bag $B_{o}$ containing $S^{\prime} \cup S^{\prime \prime}$ and connecting each one of these tree decomposition to it, we obtain a tree decomposition $\mathcal{T}$ for $R\left(S^{\prime}, C\right)$ with $f-\operatorname{tw}\left(R\left(S^{\prime}, C\right)\right) \leq f$ - width $(\mathcal{T}) \leq \max _{1 \leq i \leq t} f-\operatorname{tw}\left(R\left(S, C_{i}\right)\right)$. Since this is true for every block associated with $S^{\prime}$, we obtain a contradiction, i.e. the minimum is indeed achieved by an inclusion-minimal separator $S^{\prime}$. But then Lemma 5 (i) guarantees that $\mathcal{C}_{G}\left(S^{\prime}\right)=\mathcal{C}_{G}^{*}\left(S^{\prime}\right)$.

It remains to show how to compute $f$-optimal tree decompositions of full blocks. This is done in Lemma 10 below by using the following lemma from [BT01] (cf. Fig. 1):

Lemma 9 (Theorem 4.7 in [BT01]). Let $G$ be a graph and $(S, C)$ a full block of $G$. Then a graph $I_{R}$ is a minimal triangulation of $R(S, C)$ if and only if there is a potential maximal clique $\Omega \subseteq S \cup C$ of $G$ with $S \subset \Omega$ such that the following holds:

We have $V\left(I_{R}\right):=S \cup C$ and $E\left(I_{R}\right):=\bigcup_{i=1}^{p} E\left(I_{i}\right) \cup \Omega^{2}$, where $I_{i}$ is a minimal triangulation of $R\left(S_{i}, C_{i}\right)$ for each block $\left(S_{i}, C_{i}\right)$ associated to $\Omega$ in $R(S, C)$.

Lemma 10. Let $G$ be a graph, $(S, C)$ a full block of $G$, and $f \in \mathcal{F}(V(G))$ a width function. Then

$$
f-\operatorname{tw}(R(S, C))=\min _{\substack{\Omega \in \Pi_{G} \\ S \subset \Omega \subseteq S \cup C}} \max _{i}\left\{f(\Omega), f-\operatorname{tw}\left(R\left(S_{i}, C_{i}\right)\right)\right\}
$$

where the maximum is taken over all blocks $\left(S_{i}, C_{i}\right)$ associated to $\Omega$ in $R(S, C)$.

Proof. Let $I_{R}$ be a minimal triangulation of $R(S, C)$, that minimizes $f-\omega\left(I_{R}\right)$. By Lemma 4, we have $f-\operatorname{tw}(R(S, C))=f-\omega\left(I_{R}\right)$. Lemma 9 implies the existence of a potential maximal clique $\Omega \subseteq(S, C)$ of $G$ with $S \subset \Omega$ such that the following is true:

For each block $\left(S_{i}, C_{i}\right)$ associated to $\Omega$ in $R(S, C)$ there is a minimal triangulation $I_{i}$ of $R\left(S_{i}, C_{i}\right)$ such that $I_{R}=\left(S \cup C, E\left(I_{R}\right)\right)$ with $E\left(I_{R}\right):=\bigcup_{i=1}^{p} E\left(I_{i}\right) \cup \Omega^{2}$. Clearly $\Omega$ is a clique in $I_{R}$ and hence $f(\Omega) \leq f-\omega\left(I_{R}\right)=f$ - $\operatorname{tw}(R(S, C))$.

Now let $\left(S_{i}, C_{i}\right)$ be any block associated to $\Omega$ in $R(S, C)$. By definition, $S_{i}$ is a clique in $R\left(S_{i}, C_{i}\right)$ and therefore also in $I_{i}$ and $I_{R}$. Hence, $I_{R}\left[S_{i} \cup C_{i}\right]=I_{i}$ by definition of $E\left(I_{R}\right)$. Thus, every clique of $I_{i}$ is also a clique of $I_{R}$ and we have

$$
f-\operatorname{tw}\left(R\left(S_{i}, C_{i}\right)\right) \leq f-\omega\left(I_{i}\right) \leq f-\omega\left(I_{R}\right)=f-\operatorname{tw}(R(S, C)) .
$$

The leftmost inequality holds by Lemma 4

For the other direction let $\Omega$ be some potential maximal clique of $G$ satisfying $S \subset \Omega \subseteq S \cup C$ and define $w:=\max _{i}\left\{f(\Omega), f-\operatorname{tw}\left(R\left(S_{i}, C_{i}\right)\right)\right\}$. The existence of such an $\Omega$ is guaranteed by Lemma 9 . Let $\mathcal{T}_{i}=\left(T_{i},\left(B_{t}^{i}\right)_{t \in V\left(T_{i}\right)}\right)$ be $f$-optimal tree decompositions of $R\left(S_{i}, C_{i}\right)$. Each $S_{i}$ is a clique in $R\left(S_{i}, C_{i}\right)$. Thus there is a vertex $t_{i} \in V\left(T_{i}\right)$ with $S_{i} \subseteq B_{t_{i}}^{i}$ by Lemma 1 (i). We construct a tree decomposition $\mathcal{T}$ of $R(S, C)$ as the union of the tree decompositions $\mathcal{T}_{i}$, adding a new vertex $t$ and the new edges $\left\{t, t_{i}\right\}$. We define the bag of $t$ to be $B_{t}:=\Omega$.

The sets $C_{i}$ are the components of $R(S, C)-\Omega$. Therefore the realizations $R\left(S_{i}, C_{i}\right)$ do only intersect in the sets $S_{i} \subseteq \Omega$. Hence, every edge $e$ of $R(S, C)$ is either contained in $\Omega-$ and thus in $B_{t}$ - or belongs to one of the realizations $R\left(S_{i}, C_{i}\right)$ and so, must be contained in a bag of the tree decomposition $\mathcal{T}_{i}$. We conclude that $\mathcal{T}$ is a tree decomposition of $R(S, C)$ with $w=f$ - $\operatorname{width}(\mathcal{T}) \geq$ $f-\operatorname{tw}(R(S, C))$. 
Combining the statements of Lemmas 8 and 10 we construct Algorithm 1 . Note that Lemma 5 (iii) and Lemma 8 justify considering only full blocks in this algorithm.

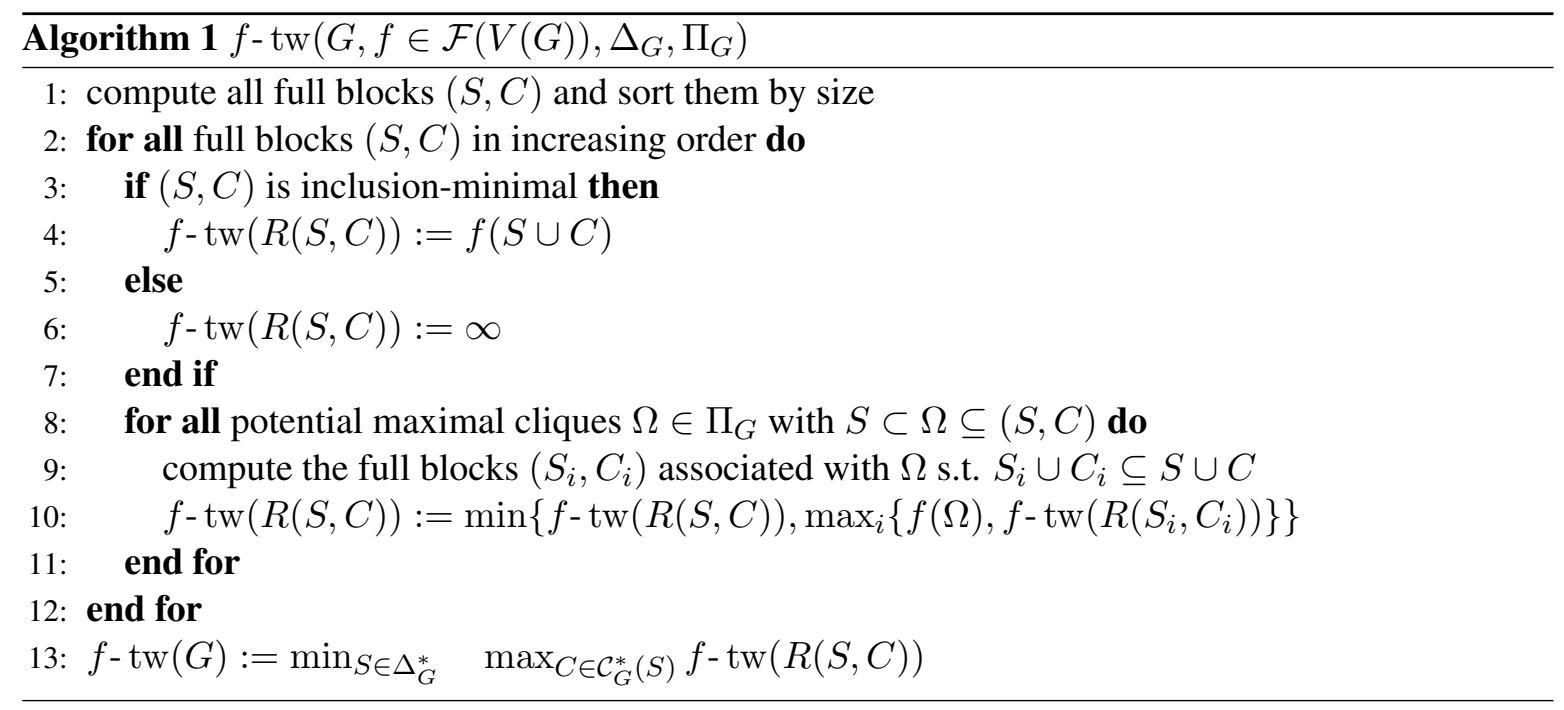

\subsection{Runtime Analysis}

As Algorithm 1] is an adaptation of the algorithm presented in [FKTV08] the runtime analysis will follow closely the analysis in that paper. However our situation necessitates a bit of preparation. Consider some input of the algorithm consisting of $f_{H}$ for some hypergraph $H=(V(H), E(H))$ and a graph $G=(V(G), E(G))$ with $V(G)=V(H)$. It will be convenient to separate the actual running time of the algorithm from the time to compute the function $f_{H}$ on all relevant subsets of $V(G)$. To this end, let a table of $f_{H}$ w.r.t. $G$ be a list of all inclusion minimal full blocks $(S, C)$ of $G$ and all potential maximal cliques $\Omega$ together with the values of $f_{H}(S \cup C)$ and $f_{H}(\Omega)$, respectively, for each of these. We obtain the following result.

Theorem 2. Let $H$ be a hypergraph with $n:=|V(H)|, m=|E(H)|$, and $f_{H} \in \mathcal{F}(V(H))$ a width function. Let $t(m, n)$ be an upper bound for the time needed to compute a table of $f_{H}$ w.r.t. the Gaifman graph $\underline{H}$. Then there is an algorithm that computes $f_{H^{-}} \mathrm{htw}(H)$ together with an $f_{H^{-}}$ optimal tree decomposition of $H$ in time $\mathcal{O}\left(1.734601^{n}+t(m, n)+m n^{2}\right)$.

The proof of this theorem readily follows from Lemma 12 below, the fact that the Gaifman graph of $H$ can be computed in time $\mathcal{O}\left(m n^{2}\right)$, and the following results of [FV08] and [FV10]:

Lemma 11 ([[FV08, FV10]). For every graph $G$ on $n$ vertices the following is true. We have $\left|\Delta_{G}\right|=$ $\mathcal{O}\left(1.6181^{n}\right)$ and $\left|\Pi_{G}\right|=\mathcal{O}\left(1.734601^{n}\right)$. Furthermore, all minimal separators and all potential maximal cliques can be listed in time $\mathcal{O}\left(1.734601^{n}\right)$.

Lemma 12. Let $G$ be a graph with $n:=|V(G)|$ and $f \in \mathcal{F}(V(G))$ a width function. Given the lists of all minimal separators $\Delta_{G}$ and of all potential maximal cliques $\Pi_{G}$ of $G$ and given a table of $f$ w.r.t. $G$, Algorithm 1 computes $f$ - $\operatorname{tw}(G)$ together with an $f$-optimal tree decomposition of $G$ in time $\mathcal{O}\left(n^{2} \cdot\left|\Delta_{G}\right|+n^{3} \cdot\left|\Pi_{G}\right|\right)$.

Proof. W.l.o.g. we assume here that the graph $G$ is connected. Otherwise we simply run the algorithm once for each connected component of $G$. The correctness of the algorithm follows easily: By 
Lemma 10 and Lemma 5 (iii) the for-loop in the lines 2, 12 correctly computes $f$ - $\operatorname{tw}(R(S, C))$ for all full blocks $(S, C)$ of $G$. Then the $f$-width of the graph is computed in line 13 using Lemma 8 . As a table of $f$ w.r.t. $G$ is given, the proof of the running time is the same as in [FKTV08].

\subsection{Computing Fractional Hypertree-Width}

Lemma 13. Let $H=(V(H), E(H))$ be a hypergraph with $n$ vertices and $m$ hyperedges. A table of $\rho_{H}^{*}$ w.r.t $\underline{H}$ can be computed in time $t(m, n)=\mathcal{O}\left(1.734601^{n} \cdot m\right)$.

Proof. Note that we can compute $\underline{H}$ from $H$ in time $\mathcal{O}\left(m n^{2}\right)$. By Lemma 11 we can construct a list of all minimal separators and all potential maximal cliques of $\underline{H}$ in time $\mathcal{O}\left(1.734601^{n}\right)$.

The list of minimal separators can be used to compute a list of all full blocks just as has been done in [FKTV08] in $\mathcal{O}\left(1.734601^{n}\right)$ time. We show how to compute the values $\rho_{H}^{*}(\Omega)$ for each potential maximal clique; the computation for full blocks works analogously. For each potential maximal clique $\Omega$ in the list, we set up the linear program

$$
\begin{array}{ll}
\text { minimize } & \sum_{e \in E(H)} \gamma_{e} \\
\text { subject to } & \sum_{e \ni v} \gamma_{e} \geq 1 \quad \text { for all } v \in \Omega .
\end{array}
$$

This takes $\mathcal{O}(m n)$ time and space. By standard facts from linear programming, we know that this program has an optimal rational solution. By a standard linear programming algorithm (see e.g. [Kar84]) this program can be solved in time $\operatorname{poly}(n) \cdot m$.

Combining this with Theorem 2, We obtain

Corollary 1. The fractional hypertree-width of a given hypergraph $H$ and a corresponding tree decomposition can be computed in time $\mathcal{O}\left(1.734601^{n} \cdot m\right)$.

\subsection{Computing Generalized Hypertree Width}

For a function $f: A \rightarrow B$, with $|A|=n$, we say that $f(x)$ can be computed in time $\mathcal{O}(g(n))$ to mean the time needed to evaluate $f$ once at input $x \in A$. We say a table of $f$ can be computed in time $\mathcal{O}\left(g^{\prime}(n)\right)$ if the value of $f(x)$ can be computed and stored in a table for every $x \in A$ in total time $\mathcal{O}\left(g^{\prime}(n)\right)$.

Let us fix a hypergraph $H$ on $n$ vertices and $m$ edges. In order to compute the generalized hypertree-width of $H$ using Theorem 2, we need to compute a table of $\rho_{H}$ (w.r.t. $\underline{H}$ ). This can be accomplished by a fairly straightforward dynamic programming algorithm in time $\mathcal{O}\left(2^{n} m n\right)$ : build a table with an entry for every pair $(U, i), 1 \leq i \leq m$, where $U$ is a subset of the vertices and $\left\{e_{1}, \ldots, e_{m}\right\}$ are the edges of the graph. For every $(U, i)$, store the minimum-size edge cover for $U$ that uses only edges $\left\{e_{1}, \ldots, e_{i}\right\}$; this can be easily done by considering the entries stored at $(U, i-1)$ and $\left(U \backslash e_{i}, i-1\right)$. An additional factor of $n$ is needed to look up an add $n$-bit integers.

This approach of computing $\rho_{H}$ has the drawback of being dependent on $m$, which itself might be exponential in $n$ an thus yields an overall running time of $O\left(n 4^{n}\right)$ in the worst case. Fortunately, as we shall see now, there is an elegant machinery which allows us to significantly improve this time bound. 


\subsubsection{Faster Computation using the Fast Möbius Transform}

Using the principle of inclusion-exclusion and the fast zeta transform, Björklund et al. [BHK09] show, for a given set $N$ of $n$ elements and a family of its subsets, how to count the number of $k$-covers of $N$ in time $\mathcal{O}\left(2^{n} n^{2}\right)$, where $k$ is part of the input. This leads to an $\mathcal{O}\left(3^{n} n^{2}\right)$-time algorithm to compute a table for $\rho_{H}^{k}$, where $\rho_{H}^{k}(U)$ denotes the number of edge covers of $U \subseteq V(H)$ using at most $k$ hyperedges. We show how to improve this running time to $\mathcal{O}\left(2^{n} n^{3}\right)$.

Let $N$ be an $n$-element set and $f: 2^{N} \rightarrow \mathbb{R}$ be a real-valued function on the set of all subsets of $N$. The zeta transform [Rot] of $f$, denoted by $\hat{f}: 2^{N} \rightarrow \mathbb{R}$ is defined as

$$
\hat{f}(Y)=\sum_{S \subseteq Y} f(S), \quad \text { for } Y \subseteq N .
$$

The straightforward method to compute a table for the zeta transform of $f$, i.e. compute $\hat{f}(Y)$ for all $Y \subseteq N$, requires $\mathcal{O}\left(3^{n}\right)$ additions in total. However, this can be improved to $\mathcal{O}\left(2^{n} n\right)$ additions using Yates's method [Yat37, BHK09] as specified in the following lemma; this algorithm is known as the fast Möbius transform or the fast zeta transform; we use the latter term in this work.

Lemma 14 ([Yat37, BHK09]). Let $N$ be a set of $n$ elements and $f: 2^{N} \rightarrow \mathbb{N}$ a function in the range $[-M, M]$. A table for the zeta transform $\hat{f}$ of $f$ can be computed via $\mathcal{O}\left(2^{n} n\right)$ additions with $\mathcal{O}(n \log M)$-bit integers.

For a set $X \subseteq V(H)$, define the number of edges that avoid $X$ as $a(X)=|\{e \in E(H) \mid e \cap X=\emptyset\}|$. Using the principle of inclusion-exclusion, Björklund et al. [BHK09] show

Lemma 15 (adapted from [BHK09]). Let $H$ be a hypergraph. For a set $U \subseteq V(H)$, let $\rho_{H}^{k}(U)$ denote the number of edge covers of $U$ using at most $k$ hyperedges; furthermore, let $a(U)$ be the number of hyperedges that avoid $U$. Then we have

$$
\rho_{H}^{k}(U)=\sum_{X \subseteq U}(-1)^{|X|} a(X)^{k} .
$$

Now we are ready to state the main result of this subsection:

Theorem 3. Let $H$ be a hypergraph on $n$ vertices and $m$ edges. Let $\rho_{H}^{k}: 2^{V(H)} \rightarrow \mathbb{N}$ be the function that counts the number of edge covers with at most $k$ hyperedges for every subset of $V(H)$. Then a table for $\rho_{H}^{k}$ can be computed in time $\mathcal{O}\left(2^{n} n^{3}\right)$.

Proof. First, we compute the values $a(X)$ for every $X \subseteq V(H)$ using the idea in [BHK09]: observe that if $e(S)$ is the indicator function telling if $S \subseteq V(H)$ is an edge or not, then

$$
a(X)=\sum_{S \subseteq V(H) \backslash X} e(S)=\hat{e}(V(H) \backslash X)
$$

can be computed from the zeta transform of $e$. Hence, a table for $g(X):=(-1)^{|X|} a(X)^{k}$ can be pre-computed and stored in time $\mathcal{O}\left(2^{n} n^{2}\right)$ using Lemma 14 (having accounted for an overhead factor of $n$ for looking up and adding $n$-bit integers). But then, Equation $\left(3\right.$ implies that $\rho_{H}^{k}$ is just the zeta-transform of $g$ and hence, a table for $\rho_{H}^{k}$ can be computed in time $\mathcal{O}\left(2^{n} n^{3}\right)$ using $\mathcal{O}\left(n^{2}\right)$-bit integers. 
For any given hypergraph $H$ and integer $k$, we can compute a table that stores for every subset $U \subseteq V(H)$ if it has an edge cover of size at most $k$ using Theorem 3 Together with Theorem 2 this implies an $\mathcal{O}\left(2^{n} n^{3}\right)$-time algorithm to decide whether the generalized hypertree-width of a given graph is at most $k$ and if so, compute a corresponding tree decomposition. The tree decomposition with the minimum generalized hypertree-width can then be obtained by binary search on $k$, adding only another factor of $n$ as overhead. Note, however, that this method does not compute the actual (minimum) edge cover for each bag of the tree decomposition; to this end, the simple dynamic programming algorithm described in the beginning of this subsection has to be used.

Corollary 2. The generalized hypertree-width of a given hypergraph $H$ and a corresponding tree decomposition can be computed in time $\mathcal{O}^{*}\left(2^{n}\right)$. The minimum edge cover for every bag of the tree decomposition can be computed in total time $\mathcal{O}^{*}\left(2^{n} m\right)$.

\section{Conclusion}

We present an algorithm that computes the $f$-width of a hypergraph for any monotone function $f$. Apart from the overhead in computing $f$, the algorithm works within the same time bound as the currently fastest exact algorithms for tree-width. As a consequence we obtain fast exact algorithms to compute the generalized and fractional hypertree-widths of a hypergraph.

An important open question is whether these algorithms can be further developed to also compute the more general hypertree width measures of adaptive width and submodular width (see Marx [Mar09b, Mar10]).

\section{References}

[BHK09] Andreas Björklund, Thore Husfeldt, and Mikko Koivisto. Set partitioning via inclusionexclusion. SIAM J. Comput., 39(2):546-563, 2009.

[BK09] Libor Barto and Marcin Kozik. Constraint satisfaction problems of bounded width. In FOCS, pages 595-603. IEEE Computer Society, 2009.

[BP93] Jean R. S. Blair and Barry Peyton. An introduction to chordal graphs and clique trees. In Graph theory and sparse matrix computation, volume 56 of IMA Vol. Math. Appl., pages 1-29. Springer, New York, 1993.

[BT01] Vincent Bouchitté and Ioan Todinca. Treewidth and minimum fill-in: Grouping the minimal separators. SIAM J. Comput., 31(1):212-232, 2001.

[Bul06] Andrei A. Bulatov. A dichotomy theorem for constraint satisfaction problems on a 3element set. J. ACM, 53(1):66-120, 2006.

[FKTV08] Fedor V. Fomin, Dieter Kratsch, Ioan Todinca, and Yngve Villanger. Exact algorithms for treewidth and minimum fill-in. SIAM J. Comput., 38(3):1058-1079, 2008.

[FV98] Tomás Feder and Moshe Y. Vardi. The computational structure of monotone monadic SNP and constraint satisfaction: A study through datalog and group theory. SIAM J. Comput., 28(1):57-104, 1998. 
[FV08] Fedor V. Fomin and Yngve Villanger. Treewidth computation and extremal combinatorics. In Luca Aceto, Ivan Damgård, Leslie Ann Goldberg, Magnús M. Halldórsson, Anna Ingólfsdóttir, and Igor Walukiewicz, editors, ICALP (1), volume 5125 of Lecture Notes in Computer Science, pages 210-221. Springer, 2008.

[FV10] Fedor V. Fomin and Yngve Villanger. Finding induced subgraphs via minimal triangulations. In STACS, pages 383-394, 2010.

[GLS02] Georg Gottlob, Nicola Leone, and Francesco Scarcello. Hypertree decompositions and tractable queries. J. Comput. Syst. Sci., 64(3):579-627, 2002.

[GM06] Martin Grohe and Dániel Marx. Constraint solving via fractional edge covers. In SODA, pages 289-298. ACM Press, 2006.

[Gro07] Martin Grohe. The complexity of homomorphism and constraint satisfaction problems seen from the other side. J. ACM, 54(1), 2007.

[GSS01] Martin Grohe, Thomas Schwentick, and Luc Segoufin. When is the evaluation of conjunctive queries tractable? In STOC, pages 657-666, 2001.

[Kar84] N. Karmarkar. A new polynomial-time algorithm for linear programming. Combinatorica, 4(4):373-395, 1984.

[KKS97] Ton Kloks, Dieter Kratsch, and Jeremy Spinrad. On treewidth and minimum fill-in of asteroidal triple-free graphs. Theor. Comput. Sci., 175(2):309-335, 1997.

[Mar09a] Dániel Marx. Approximating fractional hypertree width. In Claire Mathieu, editor, SODA, pages 902-911. SIAM, 2009.

[Mar09b] Dániel Marx. Tractable structures for constraint satisfaction with truth tables. In STACS '09: Proceedings of the 26th International Symposium on Theoretical Aspects of Computer Science, pages 649-660, 2009.

[Mar10] Dániel Marx. Tractable hypergraph properties for constraint satisfaction and conjunctive queries. In STOC, pages 735-744, 2010.

[Rot] Gian-Carlo Rota. On the foundations of combinatorial theory I. Theory of Möbius functions. Z. Wahrscheinlichkeitstheorie und Verwandte Gebiete, 2:340-368.

[Sch78] Thomas J. Schaefer. The complexity of satisfiability problems. In STOC, pages 216-226. ACM, 1978.

[Yat37] F. Yates. The design and analysis of factorial experiments. Technical Communication no. 35 of the Commonwealth Bureau of Soils, 1937. 